\title{
Physician Disengagement and Spiritual Dissonance in Medical Students
}

\author{
Cindy Schmidt, PhD; Loes Nauta, MSEd, BTE, CHSE; Andrew Dang, DO
}

\begin{abstract}
BACKGROUND AND OBJECTIVES: Religion and spirituality constitute aspects of diversity that physicians must respect to provide patient-centered care. By seeing patients as individuals and integrating their religious and spiritual needs into their medical care, providers can deliver personalized health care. Their needs become even more critical for the frontline providers during the COVID-19 pandemic. Most patients want their physicians to address their religious and spiritual needs when it comes to their health (eg, during isolation precautions). Despite increases in educational curricula about this integration, most physicians still do not provide this aspect of patientcentered care.
\end{abstract}

METHODS: In this observational study, we examined how medical students responded to a patient experiencing a religious and spiritual issue by having standardized patients (SPs) rate the students' level of engagement with them. We also asked students to reflect on their own spirituality, in terms of their current and ideal levels of spirituality, the difference of which indicates spiritual dissonance. Medical students $(n=232)$ completed the Spiritual Health and Life-Outcome Measure (SHALOM) questionnaire, and their SPs completed the Princess Margaret Hospital Satisfaction With Doctor Questionnaire (PSQ-MD).

RESULTS: Results indicated a significant, positive correlation between disengagement (from PSQ-MD) and transcendent spirituality dissonance (from SHALOM).

CONCLUSIONS: Higher levels of disconnection from a patient case with a religious and spiritual issue (portrayed by an SP) were associated with higher levels of incongruity in medical students' responses as to their ideal relationship with the transcendent (eg, God, Allah, peace).

(Fam Med. 2021;53(1):58-60.)

doi: 10.22454/FamMed.2021.194514

$\triangle$ s patient populations diversify socially and spiritually, physicians must respect their cultural diversity to provide patientcentered care. Understanding each patient's unique background and integrating patients' religious and spiritual needs into their medical care personalizes health care. ${ }^{1-3}$ Frontline providers during the COVID-19 pandemic face vastly diverse patient cultures without typical family supports (eg, isolation precautions). Even prior to the pandemic, most patients wanted their physicians to address their religious and spiritual needs, and the literature supports health benefits of this integration. ${ }^{4-9}$ Medical educators increasingly deliver curricula on addressing patients' religious and spiritual needs, with a gap, however, between education and practice. ${ }^{10-18}$ Despite receiving curricula about spirituality, few physicians integrate their patients' religious and spiritual needs into their care, returning to precurricular levels within 1 year. ${ }^{19}$ This descriptive study aimed to examine medical students' own spirituality and their ability to engage with a standardized patient (SP) presenting for a focused visit with an additional religious and spiritual issue. Specifically, we looked at students' spiritual dissonance, the state of one's currently lived spirituality as less than one's ideal spirituality. ${ }^{20-21}$

\section{Methods}

First-year, second-semester medical students (of 237 students, 5 declined consent; $\mathrm{n}=232$ ) conducted an SP interview of a focused social history in this single-institution study. To protect students' anonymity, we did not collect demographic data.

The patient in the scenario presented with the chief compliant of headache and revealed additional religious and spiritual distress due to a family member planning to marry outside her religion. Being Orthodox

From Kansas City University, Kansas City, MO (Dr Schmidt and Dang); and American University of the Caribbean School of Medicine Cupercoy, Dutch Lowlands, St. Maarten. (Ms Nauta). 
Jewish, this problem impacts the family across multiple domains: religion, family, social, and spiritual. Students were not informed prior to the interview that the SP would present this concern.

The Spiritual Health and LifeOrientation Measure (SHALOM) questionnaire contains 205 -point Likert-scored items with four subscales measuring different aspects of spiritual well-being: personal, communal, environmental, and transcendental. ${ }^{20,21}$ The individual items are rated in terms of what respondents consider ideal for spiritual well-being, as well as how they perceive their current spiritual well-being. We used mean lived and dissonance scores for each of these four types of spirituality. Half of the students completed the SHALOM after meeting with their patient (first day of the curriculum) and the other students completed the SHALOM before meeting with their patient (second day of the curriculum), so we could assess whether reflecting on their own spirituality before meeting with their patient influenced the strength of their patient engagement.

After the patient interview, SPs completed the Princess Margaret Hospital Satisfaction With Doctor
Questionnaire (PSQ-MD) about their student. ${ }^{22,23}$ The PSQ-MD contains 24 items with 5-point Likert responses that provide summative scores on two subscales: perceived support and physician disengagement. The PSQ-MD's physician disengagement subscale asks about communication, availability/accessibility, and whether patient concerns were handled with care and satisfaction. Whereas many students earned a perfect score on physician disengagement (ie, they were highly engaged with their SP), the distribution on this variable was nonnormal and unfortunately not correctable via statistical transformation. We therefore dichotomized this variable to create two subgroupings of students: engaged (total $=10-11, \mathrm{n}=125$ ) and disengaged (total=12-27, $n=107$ ), (Table 1).

We used IBM SPSS 23.0 for statistical analyses. To compare whether spirituality (ie, lived, ideal, and dissonance) in the engaged group of students differed from the disengaged group of students, we compared the groups of students using $t$ tests. To check for differences in spirituality between those students who completed the curriculum on the first vs second day of the study (ie, completing the SHALOM questionnaire after or before meeting with their patient), we compared the two groups of students (ie, students on the first vs second day) using $t$ tests. To study the relationship between engagement with patient and student spirituality, we used Pearson's correlation, calculated separately for the engaged and disengaged subgroups of students. The Institutional Review Board at American University of the Caribbean School of Medicine approved this study (\#2015-004).

\section{Results}

The response rate for this study was 97.9\%. All students who initially consented to the study completed all the study questionnaires.

$t$ tests comparing spirituality lived and spirituality dissonance were not significantly different when comparing the engaged with the disengaged students. Similarly, spirituality lived and spirituality dissonance were not significantly different for those students completing the SHALOM before meeting with their patients as compared with students completing the SHALOM after meeting with their patients.

Table 1: Descriptive Data on SHALOM and PHQ

\begin{tabular}{|c|c|c|c|c|c|c|}
\hline \multicolumn{4}{|c|}{ Engaged Students ( $n=125)$} & \multicolumn{3}{|c|}{ Disengaged Students ( $n=107)$} \\
\hline SHALOM Subscales ${ }^{1}$ & Range & Mean & SD & Range & Mean & SD \\
\hline Personal spirituality - lived & $1.4-5.0$ & 4.2 & 0.74 & $1.6-5.0$ & 4.11 & 0.71 \\
\hline Communal spirituality - lived & $2.0-5.0$ & 4.3 & 0.65 & $1.6-5.0$ & 4.24 & 0.63 \\
\hline Environmental spirituality - lived & $1.4-5.0$ & 3.4 & 0.88 & $1.0-5.0$ & 3.43 & 0.92 \\
\hline Transcendental spirituality - lived & $1.0-5.0$ & 3.34 & 1.17 & $1.0-5.0$ & 3.23 & 1.11 \\
\hline Personal spirituality - dissonance & $-1.6-2.3$ & 0.3 & 0.67 & $-1.6-2.3$ & 0.37 & 0.59 \\
\hline Communal spirituality - dissonance & $-1.4-1.9$ & 0.2 & 0.56 & $-1.4-1.8$ & 0.25 & 0.47 \\
\hline Environmental spirituality - dissonance & $-1.6-2.1$ & 0.26 & 0.56 & $-1.6-2.0$ & -0.15 & 0.60 \\
\hline Transcendental spirituality - dissonance & $-1.6-3.0$ & 0.53 & 0.81 & $-0.6-3.1$ & 0.52 & 0.76 \\
\hline \multicolumn{7}{|l|}{ PHQ Subscale ${ }^{2}$} \\
\hline Physician disengagement & $10-11$ & 10.3 & 0.47 & $12-28$ & 16.62 & 3.94 \\
\hline
\end{tabular}

Abbreviations: SHALOM, Spiritual Health and Life-Orientation Measure; PHQ, patient health questionnaire; SD, standard deviation.

1 Mean scores on spirituality were not significantly different in t tests comparing the engaged and disengaged subgroups of students.

2 Students scoring 10-11 on the PHQ (good engagement) were categorized as engaged. Students scoring $12+$ on the PHQ (somewhat to very disengaged) were categorized as disengaged. 
Among students whom their SPs rated as engaged, their score on physician disengagement was not significantly correlated with any of the spirituality subscales, for spirituality lived or for spirituality dissonance. On the contrary, among students whom their SPs rated as disengaged, their score on physician disengagement was significantly correlated with transcendental spirituality dissonance $(r=.327, P<.001)$, with a medium effect size.

\section{Discussion}

This study's results suggest that stronger spiritual dissonance (with the transcendent) is related to greater physician disengagement, in an $\mathrm{SP}$ encounter regarding religion and spirituality. That is, the greater the student's spiritual dissonance with the transcendent, the greater the student's disengagement from their SP for a patient case involving a religious and spiritual issue.

Results indicate a tendency for students to disengage from their patients when presented with a spiritual issue when the students themselves are also experiencing a spiritual issue. This could explain the low rates of physicians integrating patients' spiritual needs into their overall care. There are likely multiple factors explaining such low rates, and educators need to identify and address these to help students grow more comfortable with religious and spiritual diversity when providing patient-centered care.

Our findings are limited by using observational design, students instead of physicians, and simulation, where it is presumed that performance reflects ability. Additional limitations include using a single institution and the possibility that students may have completed the SHALOM based on what they felt instructors were looking for, as opposed to truly self-reflecting. Future research could study physicians in a nonsimulation context, examining why they engage or disengage with patients having spiritual issues. Future studies may clarify how patient spirituality impacts dissonant providers, helping to close the gap between education and practice and improving patient-centered care.

ACKNOWLedGMENTS: Melissa Patterson, MD, Adam Ellis, MD, and Sarah McCarty, MD contributed to the curriculum development and delivery of this focused social history. The authors thank the standardized patients and staff of the Clinical Skills Simulation Center at American University of the Caribbean School of Medicine for their effort learning and producing this standardized patient experience. Rabbi Moishe Chanowitz graciously gave advice on creating the Orthodox Jewish standardized patient case.

CORRSPONDING AUTHOR: Address Correspondence to Dr Cindy Schmidt, Director of Scholarly Activity and Faculty Development, Kansas City University of Medicine and Biosciences, 1750 Independence Ave, Kansas City, MO 64106. CSchmidt@kansascity.edu.

\section{References}

1. Choi PJ, Curlin FA, Cox CE. "The patient is dying, please call the chaplain": the activities of chaplains in one medical center's intensive care units. J Pain Symptom Manage. 2015;50(4):501-506

2. Williams JA, Meltzer D, Arora V, Chung G Curlin FA. Attention to inpatients' religious and spiritual concerns: predictors and association with patient satisfaction. J Gen Intern Med. 2011;26(11):1265-1271.

3. Handzo G, Koenig HG. Spiritual care: whose job is it anyway? South Med J. 2004;97(12):1242 1244

4. Brooks F, Michaelson V, King N, Inchley J, Pickett W. Spirituality as a protective health asset for young people: an international comparative analysis from three countries. Int $J$ Public Health. 2018;63(3):387-395.

5. Debnam KJ, Milam AJ, Mullen MM, Lacey K, Bradshaw CP. The moderating role of spirituality in the association between stress and substance use among adolescents: differences by gender. J Youth Adolesc. 2018;47(4):818-828.

6. Oman D, Syme SL. Weighing the evidence: what is revealed by $100+$ meta-analyses and systematic reviews of religion/spirituality and health? In: Oman D, ed. Why Religion and Spirituality Matter for Public Health, Religion, Spirituality and Health: A Social Scientific Approach. Vol 2. Cham: Springer; 2018:261-281,

7. Arutyunyan T, Odetola F, Swieringa R, Niedner M. Religion and spiritual care in pediatric intensive care unit: parental attitudes regarding physician spiritual and religious inquiry. Am J Hosp Palliat Care. 2018;35(1):28-33.

8. Jim HSL, Pustejovsky JE, Park CL, et al. Religion, spirituality, and physical health in cancer patients: A meta-analysis. Cancer. 2015;121(21):3760-3768.

9. Pearce MJ, Coan AD, Herndon JE II, Koenig HG, Abernethy AP. Unmet spiritual care needs impact emotional and spiritual well-being in advanced cancer patients. Support Care Cancer. 2012;20(10):2269-2276.
10. Lucchetti G, Goncalves LM, de Carvalho F, Daher JC, Lucchetti ALG. Incorporating and teaching spirituality into medical education: an overview of the field and possible educational strategies. In: Lucchetti G, Prieto Peres M, Damiano R, eds. Spirituality, religiousness and health: from research to practice. Cham, Switzerland: Springer; 2019:249-265,

11. Herschkopf M, Jafari N, Puchalski C. Religion and spirituality in medical education. In: Balboni MJ, Peteet JR, eds. Spirituality and religion within the culture of medicine: from evidence to practice. New York, NY: Oxford University Press; 2017:195-201. 12. VanderWeele TJ, Koenig HG. A course on religion and public health at Harvard. Am J Public Health. 2017;107(1):47-49

13. Rassoulian A, Seidman C, Löffler-Stastka H. Transcendence, religion and spirituality in medicine: medical students' point of view. Medicine (Baltimore). 2016;95(38):e4953.

14. Puchalski CM, Blatt B, Kogan M, Butler A. Spirituality and health: the development of a field. Acad Med. 2014;89(1):10-16.

15. Austin P, Macleod R, Siddall P, McSherry W, Egan R. Spiritual care training is needed for clinical and non-clinical staff to manage patients' spiritual needs. J Study Spirit. 2017;7(1):50-63.

16. Ho JQ, Nguyen CD, Lopes R, Ezeji-Okoye SC, Kuschner WG. Spiritual care in the intensive care unit: a narrative review. J Intensive Care Med. 2018;33(5):279-287.

17. Best M, Butow P, Olver I. Why do we find it so hard to discuss spirituality? A qualitative exploration of attitudinal barriers. J Clin Med. 2016;5(9):77.

18. Best M, Butow P, Olver I. Do patients want doctors to talk about spirituality? A systematic literature review. Patient Educ Couns. 2015;98(11):1320-1328.

19. Schmidt CA, Patterson MA, Ellis AM, Nauta HL. Religious and spiritual assessment: A Standardized Patient curriculum intervention. Clin Simul Nurs. 2017;13(7):314-320.

20. Fisher J. You can't beat relating with God for spiritual well-being: comparing a generic version with the original spiritual well-being questionnaire called SHALOM. Religions (Basel). 2013;4(3):325-335

21. Fisher J. Development and application of a spiritual well-being questionnaire called SHALOM. Religions (Basel). 2010;1(1):105-121.

22. Loblaw DA, Bezjak A, Singh PM, et al. Psychometric refinement of an outpatient, visitspecific satisfaction with doctor questionnaire. Psychooncology. 2004;13(4):223-234.

23. Loblaw DA, Bezjak A, Bunston T. Development and testing of a visit-specific patient satisfaction questionnaire: the Princess Margaret Hospital Satisfaction With Doctor Questionnaire. J Clin Oncol. 1999;17(6):1931-1938. 